\title{
Frailty Predicts Morbidity and Mortality After Laparoscopic Cholecystectomy for Acute Cholecystitis: An ACS-NSQIP Cohort Analysis
}

\author{
Alexander M. Fagenson ${ }^{1} \cdot$ Benjamin D. Powers ${ }^{2} \cdot$ Konstantinos A. Zorbas $^{3} \cdot$ Sunil Karhadkar $^{1} \cdot$ Andreas Karachristos $^{4}$. \\ Antonio Di Carlo ${ }^{1} \cdot$ Kwan N. Lau ${ }^{1}$
}

Received: 22 January 2020 / Accepted: 3 March 2020 / Published online: 24 March 2020

(C) 2020 The Society for Surgery of the Alimentary Tract

\begin{abstract}
Background Current guidelines recommend laparoscopic cholecystectomy be offered for patients with acute cholecystitis except those deemed as high risk. Few studies have examined the impact of frailty on outcomes for patients undergoing laparoscopic cholecystectomy. Therefore, the aim of this study was to determine the association of frailty with postoperative morbidity and mortality in patients undergoing laparoscopic cholecystectomy for acute cholecystitis.

Methods Patients undergoing laparoscopic cholecystectomy for acute cholecystectomy were identified from 2005 to 2010 in the American College of Surgeons National Surgical Quality Improvement Project (NSQIP). The Modified Frailty Index (mFI) was used a surrogate for frailty, and patients were stratified as non-frail (mFI 0$)$, low frailty (mFI 1-2), intermediate frailty (mFI 3-4) and high frailty $(\mathrm{mFI} \geq 5)$. Univariable and multivariable analyses were performed. Receiver operator curves (ROC) and an area under the curve (AUC) were generated to determine accuracy of $\mathrm{mFI}$ in predicting postoperative morbidity and mortality.

Results Of the 6898 patients undergoing laparoscopic cholecystectomy, 3245 (47\%) patients were non-frail. There were 2913 (42\%) patients with low-frailty, $649(9 \%)$ patients with intermediate frailty, and $91(2 \%)$ with high frailty. Clavien IV complications were higher for intermediate frail patients (OR 1.81, 95\% CI 1.00-3.28, $p=0.050)$ and high-frail patients (OR 4.59, 95\% CI $1.98-10.7, p<0.001$ ). Additionally, mortality was higher for patients with intermediate frailty (OR 4.69, 95\% CI 1.37-16.0, $p=0.014)$ and high frailty (OR 12.2, 95\% CI 2.67-55.5, $p=0.001)$. The $\mathrm{mFI}$ had excellent accuracy for mortality (AUC $=0.83$ ) and Clavien IV complications (AUC $=0.73$ ).

Conclusion Frailty is associated with postoperative morbidity and mortality in patients undergoing laparoscopic cholecystectomy for acute cholecystitis.
\end{abstract}

Keywords Acute cholecystitis $\cdot$ Laparoscopic cholecystectomy $\cdot$ Frailty $\cdot$ Outcomes research

\section{Introduction}

Laparoscopic cholecystectomy (LC) is the standard treatment for acute cholecystitis (AC), and is one of the most common operations performed with 460,000 cholecystectomies performed annually in the USA.' Given the high incidence of

This work was previously presented at World Congress of Surgery. Basel, Switzerland 2017.

Kwan N. Lau

Kwan.lau@tuhs.temple.edu

1 Department of Surgery, Lewis Katz School of Medicine-Temple University, Zone C 640, 3401 N.Board St., Philadelphia, PA 19140, USA
AC in the US population, many patients present with multiple comorbidities are at risk for complications. For these higher risk patients, operative mortality rates for LC approach $20 \%$. Gallbladder drainage in the form of a percutaneous cholecystotomy tube has been recommended as an acceptable option for these higher risk patients. ${ }^{3}$ Newer technologies

2 Department of Gastrointestinal Oncology, H. Lee Moffitt Cancer Center \& Research Institute, Tampa, FL, USA

3 Department of Surgery, BronxCare Health System, New York, NY, USA

4 Department of Surgery, University of South Florida, Tampa, FL, USA 
including endoscopic ultrasound-guided drainage and cryoablation are now being offered.

While guidelines have often characterized high-risk patients as those who are critically ill and/or elderly, few studies have examined the impact of frailty among patients undergoing laparoscopic cholecystectomy. ${ }^{2,8-11}$ Earlier version of the 2013 Tokyo guidelines focused on clinically related characteristics of cholecystitis. The 2018 revisions of Tokyo guidelines incorporated the Charlson Comorbidity Index (CCI) and American Society of Anesthesiologists (ASA) classification to account for patient-specific factors. ${ }^{12}$ Conventional risk assessment scores, such as ASA score, Model for End-stage-Liver Disease (MELD) score, Acute Physiology and Chronic Health Evaluation II (APACHE II) score, Albumin-Bilirubin score (ALBI), and Child-Pugh-Turcotte (CPT) score are of limited use. They either include limited number of preoperative factors, focus on a single organ state, or were constructed for other purposes. Thus, there is a need to identify preoperative clinical variables for high-risk patients with acute cholecystitis undergoing cholecystectomy that encompass the concept of frailty.

Frailty is an established method used to study outcomes in surgery. One of the most widely used frailty assessment tools is the frailty index from the Canadian Study on Health and Aging (CSHA). ${ }^{13}$ In 2013, Velanovich described a simplified edition of the CSHA frailty index, which was named the Modified Frailty Index (mFI). ${ }^{14}$ The $\mathrm{mFI}$ was created by mapping the variables in the American College of Surgeons National Surgical Quality Improvement Project (NSQIP) database used to calculate the CSHA score. Several studies have shown that frailty is predictive of morbidity and prolonged postoperative recovery. ${ }^{11,15-19}$ Few studies have been done to evaluate the role of $\mathrm{mFI}$ for preoperative risk assessment of patients with cholecystitis. Therefore, the aim of this study was to determine if the $\mathrm{mFI}$ was associated with postoperative morbidity and mortality in patients undergoing laparoscopic cholecystectomy for acute cholecystitis.

\section{Methods}

Patients undergoing non-emergent laparoscopic cholecystectomy from 2005 to 2010 for acute or acute-on-chronic cholecystitis were identified in the American College of Surgeons National Surgical Quality Improvement Program (ACS NSQIP) participant use file at the national level. The ACS NSQIP is a nationally validated, risk-adjusted, outcomesbased program that collects data on a prospective systematic sample of patients undergoing major surgery. Patients were identified based on Current Procedural Terminology (CPT) codes and International Classification of Disease (ICD-9) codes. Primary CPT codes utilized were 47562, 47563, 47564, 47600, 47605, and 47610, and a secondary CPT code: 47579 was present in the cases of CPT 47600 and 47610 (both "removal of gallbladder"). The ICD-9 code: 575.12 was used to identify patients with cholecystitis.

\section{Modified Frailty Index Calculation}

The mFI consists of 11 NSQIP preoperative variables. One point was allotted for each of the following preoperative comorbidities: (1) patient functional status; (2) diabetes mellitus treated with insulin or oral medications; (3) hypertension requiring treatment; (4) congestive heart failure (CHF); (5) myocardial infarction (MI); (6) prior cardiac surgery or percutaneous coronary angioplasty, or history of angina; (7) chronic obstructive pulmonary disease (COPD) or pneumonia, (8) rest pain or gangrene secondary to peripheral vascular disease (PVD) or PVD treated with angioplasty, revascularization, amputation; (9) impaired sensorium within $48 \mathrm{~h}$ prior to the surgical procedure not in the context of concomitant neurologic disease such as dementia; (10) history of transient ischemic attack (TIA) or cerebrovascular accident (CVA) without neurologic deficits; and (11) CVA with neurologic deficits. Possible $\mathrm{mFI}$ values range from 0 to 11 . Total $\mathrm{mFI}$ was calculated, and patients were stratified based on their $\mathrm{mFI}$ score and classified as non-frail ( $\mathrm{mFI} 0)$, low frailty ( $\mathrm{mFI} 1-2)$, intermediate frailty $(\mathrm{mFI} 3-4)$, or high frailty $(\mathrm{mFI} \geq 5)$ (Table 1$)$.

\section{Population Baseline Characteristics}

Baseline demographic, laboratory, and clinical data were compared. Patients' demographic variables included age, gender, race (categorized as white or other) and body mass index (BMI), and ASA score. Preoperative laboratory variables analyzed were serum albumin, total serum bilirubin, serum glutamic-oxaloacetic transaminase (SGOT), creatinine, platelet count, hematocrit, and white blood cell count.

\section{Outcomes}

Postoperative outcomes assessed included 30-day mortality and standard 30-day postoperative outcomes as routinely collected by NSQIP including superficial surgical site infection (SSI), deep SSI surgical site infection, organ space SSI surgical site infection, sepsis, septic shock, myocardial infarction (MI), cardiac arrest requiring cardiopulmonary resuscitation (CPR), pneumonia, reintubation, ventilator dependence (failure to wean from mechanical ventilation $>48 \mathrm{~h}$ after surgery), progressive renal insufficiency with no requirement for dialysis, acute renal failure with need of dialysis, urinary tract infection, deep venous thrombosis, stroke, length of stay, and operative time. Clavien class IV complications are lifethreatening complications that lead to single-organ or multiorgan dysfunction, requiring intensive care unit (ICU) management. In our study, Clavien class IV complications are any of the following complications in the NSQIP database: cardiac 
Table 1 Demographics of the study groups

\begin{tabular}{|c|c|c|c|c|c|}
\hline & No frailty $(n=3245)$ & Low frailty $(n=2913)$ & Intermediate frailty $(n=649)$ & High frailty $(n=91)$ & $p$ value \\
\hline Age, years, median (IQR) & $42(31-54)$ & $63(52-74)$ & $72(63-79)$ & $72(66-78)$ & $<0.001$ \\
\hline Male, $n(\%)$ & $1112(34)$ & $1498(51)$ & $435(67)$ & $60(66)$ & $<0.001$ \\
\hline White, $n(\%)$ & $1458(45)$ & $1387(48)$ & $305(47)$ & $38(42)$ & 0.149 \\
\hline Body mass index, $\mathrm{kg} / \mathrm{m}^{2}$ & $28.3(24.7-33.0)$ & $29.6(25.6-33.9)$ & $28.5(24.8-33.4)$ & $29.2(25.4-34.6)$ & $<0.001$ \\
\hline $\mathrm{ASA} \geq 3, n(\%)$ & $431(13)$ & $1567(57)$ & $605(93)$ & $87(96)$ & $<0.001$ \\
\hline Albumin $\leq 3.5 \mathrm{~g} / \mathrm{dl}, n(\%)$ & $732(28)$ & $1113(46)$ & $396(68)$ & $68(81)$ & $<0.001$ \\
\hline Bilirubin $\geq 1.2 \mathrm{mg} / \mathrm{dl}, n(\%)$ & $601(20)$ & $825(30)$ & $188(31)$ & $32(36)$ & $<0.001$ \\
\hline $\mathrm{SGOT} \geq 40 \mathrm{U} / \mathrm{L}$ & $834(28)$ & $788(29)$ & $196(32)$ & $38(43)$ & 0.006 \\
\hline Creatinine $\geq 1.2 \mathrm{mg} / \mathrm{dl}, n(\%)$ & $185(6.2)$ & $659(24)$ & $291(46)$ & $56(62)$ & $<0.001$ \\
\hline Platelet $\leq 150, n(\%)$ & $186(6.0)$ & $369(13)$ & $117(19)$ & $21(23)$ & $<0.001$ \\
\hline Hematocrit $\leq 35, n(\%)$ & $638(21)$ & $895(32)$ & $322(51)$ & $69(76)$ & $<0.001$ \\
\hline White blood cell $\geq 11$ & $1088(35)$ & $1138(40)$ & $302(48)$ & $50(55)$ & $<0.001$ \\
\hline \multicolumn{6}{|c|}{ Preoperative variables included in calculation of $\mathrm{mFI}$} \\
\hline Dependent functional status, $n(\%)$ & $0(0)$ & $261(9.0)$ & $275(42)$ & $78(86)$ & $<0.001$ \\
\hline Diabetes mellitus, $n(\%)$ & $0(0)$ & $662(23)$ & $402(62)$ & $75(83)$ & $<0.001$ \\
\hline Hypertension, $n(\%)$ & $0(0)$ & $2392(82)$ & $610(94)$ & $87(96)$ & $<0.001$ \\
\hline $\mathrm{CHF}, n(\%)$ & $0(0)$ & $24(0.8)$ & $37(5.7)$ & $27(30)$ & $<0.001$ \\
\hline History of MI within 6 months, $n(\%)$ & $0(0)$ & $13(0.4)$ & $35(5.4)$ & $14(15)$ & $<0.001$ \\
\hline History of PCI/CS/Angina, $n(\%)$ & $0(0)$ & $218(7.5)$ & $193(30)$ & $31(34)$ & $<0.001$ \\
\hline COPD or pneumonia, $n(\%)$ & $0(0)$ & $136(4.7)$ & $132(20)$ & $29(32)$ & $<0.001$ \\
\hline $\mathrm{PVD}, n(\%)$ & $0(0)$ & $19(0.7)$ & $60(9.2)$ & $27(30)$ & $<0.001$ \\
\hline Impaired sensorium, $n(\%)$ & $0(0)$ & $18(0.6)$ & $45(6.9)$ & $25(28)$ & $<0.001$ \\
\hline TIA/CVA without residual deficit, $n(\%)$ & $0(0)$ & $71(2.4)$ & $70(11)$ & $23(25)$ & $<0.001$ \\
\hline CVA/stroke with residual deficit, $n(\%)$ & $0(0)$ & $42(1.4)$ & $94(15)$ & $42(46)$ & $<0.001$ \\
\hline
\end{tabular}

No frailty $=m F I 0$, Low frailty $=m F I 1-2$, Intermediate frailty $=m F I 3-4$, High frailty $=m F I \geq 5$

ASA American Society of Anesthesiologists, $S G O T$ serum glutamic-oxaloacetic transaminase, $C H F$ congestive heart failure, $M I$ myocardial infarction, $P C I$ percutaneous coronary intervention, $C S$ cardiac surgery, COPD chronic obstructive pulmonary disease, $P V D$ peripheral vascular disease, TIA transient ischemic attack, CVA cerebrovascular accident; italic font represents statistically significant associations

arrest requiring CPR, MI, pulmonary embolism, postoperative acute renal failure requiring dialysis, septic shock, failure to wean from mechanical ventilation $>48 \mathrm{~h}$ after surgery, and unplanned postoperative reintubation.

\section{Statistical Analysis}

Categorical variables were expressed as frequencies $(n)$ with percentages, and continuous variables were expressed as medians with interquartile range (IQR). Normality of continuous data was measured by the Kolgomorov-Smirnov tests and continuous data was of non-normal distribution. Univariable analysis was performed by the chi square and Mann-Whitney $U$ tests for categorical and continuous variables respectively. A backward stepwise multivariable logistic regression analysis was used to identify risk factors for mortality and Clavien class IV complications. Factors that were significant on univariable analysis $(p<0.05)$ were included in the multivariable model. The impact of each individual frailty component was studied, and the odds of mortality and Clavien class IV complications were calculated for each $\mathrm{mFI}$ component. Results from multivariable analysis were reported as odds ratios (OR), 95\% confidence interval (95\% CI), and $p$ value. Receiver operator characteristic curves (ROC) and an area under the curve (AUC) were generated to measure accuracy of $\mathrm{mFI}$ in predicting mortality and Clavien class IV complications. Statistical analysis was performed using SPSS version 26 (IBM). Two-tailed $p$ values of less than 0.05 were deemed statistically significant. The study used de-identified data and was exempt from the Temple University Hospital Institutional Review Board.

\section{Results}

\section{Demographics}

A total of 6898 patients underwent laparoscopic cholecystectomy for acute cholecystitis from 2005 to 2010 . The 
preoperative characteristics for each group are shown in Table 1 . The median age was 55 years (IQR 40-69), 55\% were female $(n=3793)$, and the median BMI was $28.7 \mathrm{~kg} /$ $\mathrm{m}^{2}$ (IQR 25.1-33.4). 3245 (47\%) patients were non-frail (mFI 0), 2913 (42\%) patients with low frailty (mFI 1-2), $649(9 \%)$ with intermediate frailty (mFI 3-4), and $91(2 \%)$ high frailty $(\mathrm{mFI} \geq 5)$ patients. Increasing age, male gender, ASA score $\geq 3$, hypoalbuminemia, hyperbilirubinemia, elevated SGOT, elevated creatinine, thrombocytopenia, low hematocrit, and elevated WBC count all significantly increased as frailty increased $(p<0.001)$ (Table 1$)$. The individual frailty components as calculated in the mFI are also illustrated in Table 1.

\section{Postoperative Outcomes}

Postoperative mortality and morbidity classified by frailty groups are shown in Table 2. An increasing rate of morality and Clavien class IV complications were observed as frailty increased. For the intermediate frailty group, the mortality rate was $6.0 \%(p<0.001)$ and Clavien IV complication rate was $11 \%(p<0.001)$ (Table 2$)$. The mortality rate for the high frailty group was $20 \%(p<0.001)$ and rate of Clavien IV complications was $28 \%(p<0.001)$ (Table 2$)$. Length of stay and operative time were significantly longer as frailty increased (both $p<0.001$ ).

Univariable and multivariable regression analyses for postoperative Clavien class IV (CLIVCs) complications are shown in Table 3. After multivariable regression, intermediate and high frailty were significantly associated with an increased risk for postoperative CLIVCs (OR 1.81, 95\% CI $1.00-3.28, p=0.050$ and OR 4.59, 95\% CI 1.98-10.7, $p<0.001$ ) (Table 3 ). An ASA $\geq 3$ placed patients at increased risk (OR 3.34, 95\% CI 1.72-6.50, $p<0.001$ ) for CLIVCs; however, age $\geq 65$ years (OR $1.16,95 \% \mathrm{CI}$ $0.58-2.29, p=0.551$ ) and male sex (OR 0.95, 95\% CI $0.52-1.72, p=0.860)$ were not associated with an increased risk of CLIVCs.

Univariable and multivariable regression analyses for postoperative mortality are shown in Table 4. Intermediate and high frailty patients were at increased risk for postoperative mortality (OR 4.69, 95\% CI 1.37-16.0, $p=0.014$ and OR $12.2,95 \%$ CI 2.67-55.5, $p=0.001)$. While low frailty (mFI $1-2$ ), age $\geq 65$ years, male sex, $\mathrm{ASA} \geq 3$, albumin, bilirubin, SGOT, creatinine, WBC, and hematocrit were not associated with an increased risk for mortality.

An analysis of the adjusted risk of each frailty component toward CLIVCs is shown in Table 5. After multivariable regression, dependent functional status (OR 4.28, 95\% CI 3.21-

Table 2 30-day postoperative outcomes

\begin{tabular}{|c|c|c|c|c|c|}
\hline & No frailty $(n=3245)$ & Low frailty $(n=2913)$ & Intermediate frailty $(n=649)$ & High frailty $(n=91)$ & $p$ value \\
\hline Mortality, $n(\%)$ & $6(0.2)$ & $38(1.3)$ & $39(6.0)$ & $18(20)$ & $<0.001$ \\
\hline Clavien IV complications, $n(\%)$ & $48(1.5)$ & $156(5.4)$ & $73(11)$ & $25(28)$ & $<0.001$ \\
\hline Superficial SSI, $n(\%)$ & $37(1.1)$ & $65(2.2)$ & $18(2.8)$ & $4.0(4.4)$ & $<0.001$ \\
\hline Deep incisional SSI, $n(\%)$ & $7.0(0.2)$ & $12(0.4)$ & $6.0(0.9)$ & $4.0(4.4)$ & $<0.001$ \\
\hline Organ space SSI, $n(\%)$ & $28(0.9)$ & $45(1.5)$ & $11(1.7)$ & $2.0(2.2)$ & 0.052 \\
\hline Pneumonia, $n(\%)$ & $11(0.3)$ & $54(1.9)$ & $31(4.8)$ & $6.0(6.6)$ & $<0.001$ \\
\hline Reintubation, $n(\%)$ & $11(0.3)$ & $54(1.9)$ & $31(4.8)$ & $8.0(8.8)$ & $<0.001$ \\
\hline Pulmonary embolism, $n(\%)$ & $4.0(0.1)$ & $11(0.4)$ & $3(0.5)$ & $0(0)$ & 0.162 \\
\hline Deep venous thrombosis, $n(\%)$ & $5.0(0.2)$ & $9.0(0.3)$ & $7.0(1.1)$ & $1.0(1.1)$ & 0.001 \\
\hline Ventilator dependence, $n(\%)$ & $5.0(0.2)$ & $55(1.9)$ & 49 (7.6) & $16(18)$ & $<0.001$ \\
\hline Progressive renal insufficiency, $n(\%)$ & $3.0(0.1)$ & $28(1.0)$ & $11(1.7)$ & $9.0(9.9)$ & $<0.001$ \\
\hline Acute renal failure, $n(\%)$ & $3.0(0.1)$ & $18(0.6)$ & $10(1.5)$ & $7.0(7.7)$ & $<0.001$ \\
\hline Urinary tract infection, $n(\%)$ & $12(0.4)$ & $42(1.4)$ & $12(1.8)$ & $2(2.2)$ & $<0.001$ \\
\hline Stroke, $n(\%)$ & $0(0)$ & $6.0(0.2)$ & $6.0(0.9)$ & $1.0(1.1)$ & $<0.001$ \\
\hline Cardiac arrest requiring $\mathrm{CPR}, n(\%)$ & $30(0.1)$ & $8.0(0.3)$ & $10(1.5)$ & $7.0(7.7)$ & $<0.001$ \\
\hline Myocardial infarction, $n(\%)$ & $2.0(0.1)$ & $13(0.4)$ & $13(2.0)$ & $0(0)$ & $<0.001$ \\
\hline Sepsis, $n(\%)$ & $29(0.9)$ & $66(2.3)$ & $23(3.5)$ & $8.0(8.8)$ & $<0.001$ \\
\hline Septic shock, $n(\%)$ & $12(0.4)$ & $39(1.3)$ & $28(4.3)$ & $8.0(8.8)$ & $<0.001$ \\
\hline Reoperation, $n(\%)$ & $37(1.1)$ & $81(2.8)$ & $34(5.2)$ & $8.0(8.8)$ & $<0.001$ \\
\hline Length of stay, days, median (IQR) & $2(1-4)$ & $4(2-6)$ & $7(4-11)$ & $13(7-23)$ & $<0.001$ \\
\hline Operative time, minutes, median (IQR) & $75(52-104)$ & $84(59-115)$ & $84(63-118)$ & $87(68-112)$ & $<0.001$ \\
\hline
\end{tabular}

No frailty $=\mathrm{mFI} 0$, Low frailty $=\mathrm{mFI} 1-2$, Intermediate frailty $=\mathrm{mFI} 3-4$, High frailty $=\mathrm{mFI} \geq 5$; SSI, surgical site infection; italic font represents statistically significant associations 
Table 3 Factors associated with Clavien IV complications

\begin{tabular}{|c|c|c|c|c|c|c|}
\hline & \multicolumn{3}{|c|}{ Univariable } & \multicolumn{3}{|c|}{ Multivariable } \\
\hline & OR & $95 \% \mathrm{CI}$ & $p$ value & OR & $95 \% \mathrm{CI}$ & $p$ value \\
\hline \multicolumn{7}{|l|}{ Frailty } \\
\hline No frailty & Ref & & & Ref & & \\
\hline Low frailty & 3.77 & $2.72-5.23$ & $<0.001$ & 1.73 & $1.15-2.61$ & 0.009 \\
\hline Intermediate frailty & 8.44 & $5.80-12.28$ & $<0.001$ & 1.81 & $1.00-3.28$ & 0.050 \\
\hline High frailty & 25.2 & $14.7-43.4$ & $<0.001$ & 4.59 & $1.98-10.7$ & $<0.001$ \\
\hline Age $\geq 65$ years & 2.40 & $1.91-3.03$ & $<0.001$ & 1.16 & $0.58-2.29$ & 0.551 \\
\hline Male (vs female) & 1.70 & $1.35-2.15$ & $<0.001$ & 0.95 & $0.52-1.72$ & 0.860 \\
\hline $\mathrm{BMI} \geq 30$ & 1.16 & $0.92-1.47$ & 0.203 & - & & \\
\hline White (vs others) & 0.84 & $0.66-1.06$ & 0.138 & - & & \\
\hline $\mathrm{ASA} \geq 3, n(\%)$ & 6.31 & $4.75-8.40$ & $<0.001$ & 3.34 & $1.72-6.50$ & $<0.001$ \\
\hline Albumin $\leq 3.5 \mathrm{~g} / \mathrm{dl}, n(\%)$ & 3.23 & $2.49-4.19$ & $<0.001$ & 1.86 & $1.00-3.49$ & 0.051 \\
\hline Bilirubin $\geq 1.2 \mathrm{mg} / \mathrm{dl}, n(\%)$ & 1.79 & $1.40-2.28$ & $<0.001$ & 1.15 & $0.61-2.18$ & 0.663 \\
\hline $\mathrm{SGOT} \geq 40 \mathrm{U} / \mathrm{L}$ & 1.75 & $1.38-2.23$ & $<0.001$ & 1.16 & $0.65-2.08$ & 0.617 \\
\hline Creatinine $\geq 1.2 \mathrm{mg} / \mathrm{dl}, n(\%)$ & 3.52 & $2.77-4.49$ & $<0.001$ & 1.36 & $0.64-2.90$ & 0.420 \\
\hline Platelet $\leq 150, n(\%)$ & 2.11 & $1.56-2.86$ & $<0.001$ & 0.77 & $0.33-1.81$ & 0.547 \\
\hline Hematocrit $\leq 35, n(\%)$ & 2.79 & $2.21-3.54$ & $<0.001$ & 1.47 & $0.78-2.80$ & 0.238 \\
\hline White blood cell $\geq 11$ & 2.00 & $1.58-2.53$ & $<0.001$ & 1.31 & $0.75-2.30$ & 0.339 \\
\hline
\end{tabular}

No frailty $=m F I 0$, Low frailty $=m F I 1-2$, Intermediate frailty $=m F I 3-4$, High Frailty $=m F I \geq 5$

$B M I$ body mass index, ASA American Society of Anesthesiologists, SGOT, serum glutamic-oxaloacetic transaminase; italic font represents statistically significant associations

Table 4 Factors associated with mortality

\begin{tabular}{|c|c|c|c|c|c|c|}
\hline & \multicolumn{3}{|c|}{ Univariable } & \multicolumn{3}{|c|}{ Multivariable } \\
\hline & OR & $95 \% \mathrm{CI}$ & $p$ value & OR & $95 \% \mathrm{CI}$ & $p$ value \\
\hline \multicolumn{7}{|l|}{ Frailty } \\
\hline No frailty & Ref & & & Ref & & \\
\hline Low frailty & 7.14 & $3.01-16.9$ & $<0.001$ & 1.44 & $0.51-4.10$ & 0.494 \\
\hline Intermediate frailty & 34.5 & $14.6-81.9$ & $<0.001$ & 4.69 & $1.37-16.0$ & 0.014 \\
\hline High frailty & 133.1 & $51.4-345.1$ & $<0.001$ & 12.2 & $2.67-55.5$ & 0.001 \\
\hline Age $\geq 65$ years & 5.20 & $3.40-7.96$ & $<0.001$ & 2.24 & $0.62-8.07$ & 0.217 \\
\hline Male (vs female) & 1.59 & $1.07-2.37$ & 0.020 & 0.77 & $0.26-2.33$ & 0.647 \\
\hline $\mathrm{BMI} \geq 30$ & 0.70 & $0.46-1.06$ & 0.091 & - & & \\
\hline White (vs others) & 1.06 & $0.71-1.56$ & 0.790 & - & & \\
\hline $\mathrm{ASA} \geq 3, n(\%)$ & 29.4 & $11.9-72.4$ & $<0.001$ & 6.04 & $0.99-36.89$ & 0.052 \\
\hline Albumin $\leq 3.5 \mathrm{~g} / \mathrm{dl}, n(\%)$ & 7.15 & $4.09-12.51$ & $<0.001$ & 1.43 & $0.38-5.38$ & 0.594 \\
\hline Total bilirubin $\geq 1.2 \mathrm{mg} / \mathrm{dl}, n(\%)$ & 3.88 & $2.58-5.85$ & $<0.001$ & 1.73 & $0.57-5.22$ & 0.330 \\
\hline $\mathrm{SGOT} \geq 40 \mathrm{U} / \mathrm{L}$ & 2.81 & $1.87-4.25$ & $<0.001$ & 1.98 & $0.65-6.01$ & 0.228 \\
\hline Creatinine $\geq 1.2, n(\%)$ & 5.46 & $3.65-8.17$ & $<0.001$ & 1.26 & $0.38-4.19$ & 0.712 \\
\hline Platelet $\leq 150, n(\%)$ & 4.03 & $2.62-6.22$ & $<0.001$ & 1.03 & $0.27-3.88$ & 0.966 \\
\hline Hematocrit $\leq 35, n(\%)$ & 5.30 & $3.47-8.10$ & $<0.001$ & 1.44 & $0.38-5.50$ & 0.591 \\
\hline White blood cell $\geq 11$ & 1.71 & $1.15-2.55$ & 0.008 & 1.99 & $0.68-5.81$ & 0.209 \\
\hline
\end{tabular}

No frailty $=\mathrm{mFI} 0$, Low frailty $=\mathrm{mFI} 1-2$, Intermediate frailty $=\mathrm{mFI} 3-4$, High frailty $=\mathrm{mFI} \geq 5$

$B M I$ body mass index, ASA American Society of Anesthesiologists, SGOT, serum glutamic-oxaloacetic transaminase; italic font represents statistically significant associations 
Table 5 Individual frailty components associated with Clavien IV complications

\begin{tabular}{|c|c|c|c|c|c|c|}
\hline & \multicolumn{3}{|c|}{ Univariable } & \multicolumn{3}{|c|}{ Multivariable } \\
\hline & OR & $95 \% \mathrm{CI}$ & $p$ value & OR & $95 \% \mathrm{CI}$ & $p$ value \\
\hline Dependent functional status (vs independent) & 6.49 & $5.03-8.35$ & $<0.001$ & 4.28 & $3.21-5.71$ & $<0.001$ \\
\hline Diabetes mellitus & 2.65 & $2.07-3.40$ & $<0.001$ & 1.42 & $1.07-1.87$ & 0.013 \\
\hline Hypertension & 3.61 & $2.78-4.68$ & $<0.001$ & 2.45 & $1.85-3.25$ & $<0.001$ \\
\hline Congestive heart failure & 5.49 & $3.19-9.43$ & $<0.001$ & 1.80 & $0.97-3.31$ & 0.061 \\
\hline History of MI within 6 months & 3.30 & $1.56-6.99$ & 0.001 & 1.36 & $0.59-3.12$ & 0.471 \\
\hline History of $\mathrm{PCI} / \mathrm{CS} /$ angina & 1.99 & $1.38-2.87$ & $<0.001$ & 1.07 & $0.62-1.60$ & 0.726 \\
\hline COPD or pneumonia & 3.40 & $2.36-4.91$ & $<0.001$ & 1.80 & $1.21-2.68$ & 0.004 \\
\hline Peripheral vascular disease & 3.15 & $1.74-5.69$ & $<0.001$ & 1.09 & $0.57-2.07$ & 0.791 \\
\hline Impaired sensorium & 5.48 & $3.19-9.43$ & $<0.001$ & 1.55 & $0.85-2.83$ & 0.155 \\
\hline TIA/CVA without residual deficit & 2.80 & $1.69-4.63$ & $<0.001$ & 1.38 & $0.80-2.36$ & 0.244 \\
\hline CVA/stroke with residual deficit & 3.07 & $1.91-4.91$ & $<0.001$ & 0.86 & $0.51-1.44$ & 0.561 \\
\hline
\end{tabular}

$M I$ myocardial infarction, $P C I$ percutaneous coronary intervention, $C S$ cardiac surgery, $C O P D$ chronic obstructive pulmonary disease, $T I A$ transient ischemic attack, CVA cerebrovascular accident; italic font represents statistically significant associations

$5.71, p<0.001$ ), hypertension (OR 2.45, 95\% CI 1.85-3.21, $p<0.001$ ), COPD or pneumonia preoperatively (OR 1.80 , 95\% CI 1.21-2.68, $p=0.004$ ), and diabetes mellitus (OR $1.42,95 \%$ CI $1.07-1.87, p=0.013$ ) placed patients at increased risk for postoperative CLIVCs (Table 5).

An analysis of the adjusted risk of each frailty component toward mortality is shown in Table 6. Dependent functional status (OR 9.28, 95\% CI 5.81-14.8, $p<0.001$ ), hypertension (OR 1.83, 95\% CI 1.09-3.06, $p=0.023$ ), congestive heart failure (OR 2.72, 95\% CI 1.27-5.84, $p=0.010$ ), history of $\mathrm{PCI} / \mathrm{CS} /$ Angina (OR 2.03, 95\% CI 1.25-3.30, $p=0.004$ ), COPD or pneumonia (OR 3.11, 95\% CI 1.87-5.17, $p<0.001$ ), and impaired sensorium (OR 4.34, 95\% CI 2.25-
$8.38, p<0.001)$ were all significantly associated with an increased risk for postoperative mortality (Table 6).

Receiver operator characteristic curves (ROC) demonstrate that the $\mathrm{mFI}$ can accurately predict mortality and CLVCs in this patient cohort. The area under the curve (AUC) for mortality was 0.83 , while the AUC for CLIVCs was 0.73 (Fig. 1).

\section{Discussion}

In this analysis, we show that frailty as measured by $\mathrm{mFI}$ is associated with increased postoperative morbidity and mortality for patients undergoing laparoscopic cholecystectomy for

Table 6 Individual frailty components associated with mortality

\begin{tabular}{|c|c|c|c|c|c|c|}
\hline & \multicolumn{3}{|c|}{ Univariable } & \multicolumn{3}{|c|}{ Multivariable } \\
\hline & OR & $95 \% \mathrm{CI}$ & $p$ value & OR & $95 \% \mathrm{CI}$ & $p$ value \\
\hline Dependent functional status (vs Independent) & 18.0 & $11.9-27.1$ & $<0.001$ & 9.28 & $5.81-14.8$ & $<0.001$ \\
\hline Diabetes mellitus & 3.12 & $2.08-4.70$ & $<0.001$ & 1.26 & $0.78-2.04$ & 0.341 \\
\hline Hypertension & 4.03 & $2.54-6.39$ & $<0.001$ & 1.83 & $1.09-3.06$ & 0.023 \\
\hline Congestive heart failure & 14.6 & $7.95-26.9$ & $<0.001$ & 2.72 & $1.27-5.84$ & 0.010 \\
\hline History of MI within 6 months & 4.79 & $1.71-13.5$ & 0.001 & 0.99 & $0.29-3.39$ & 0.990 \\
\hline History of PCI/CS/angina & 2.19 & $1.21-3.95$ & 0.008 & 2.03 & $1.25-3.30$ & 0.004 \\
\hline COPD or pneumonia & 8.18 & $5.20-12.8$ & $<0.001$ & 3.11 & $1.87-5.17$ & $<0.001$ \\
\hline Peripheral vascular disease & 2.71 & $0.98-7.50$ & 0.046 & 0.48 & $0.15-1.50$ & 0.204 \\
\hline Impaired sensorium & 20.8 & $11.9-36.5$ & $<0.001$ & 4.34 & $2.25-8.38$ & $<0.001$ \\
\hline TIA/CVA without residual deficit & 3.15 & $1.44-6.90$ & 0.002 & 1.59 & $0.63-4.03$ & 0.328 \\
\hline $\mathrm{CVA} /$ stroke with residual deficit & 4.34 & $2.22-8.48$ & $<0.001$ & 0.66 & $0.31-1.43$ & 0.295 \\
\hline
\end{tabular}

$M I$ myocardial infarction, $P C I$ percutaneous coronary intervention, $C S$ cardiac surgery, $C O P D$ chronic obstructive pulmonary disease, TIA transient ischemic attack, $C V A$ cerebrovascular accident; italic font represents statistically significant associations 
a

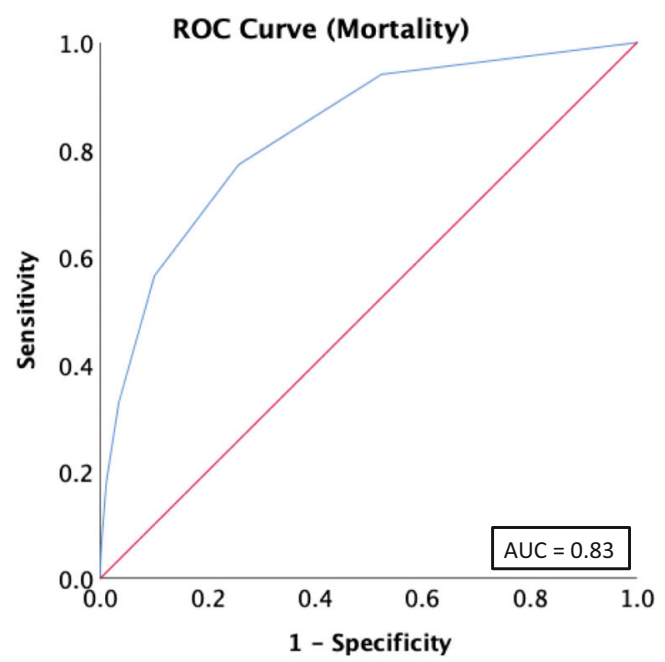

b

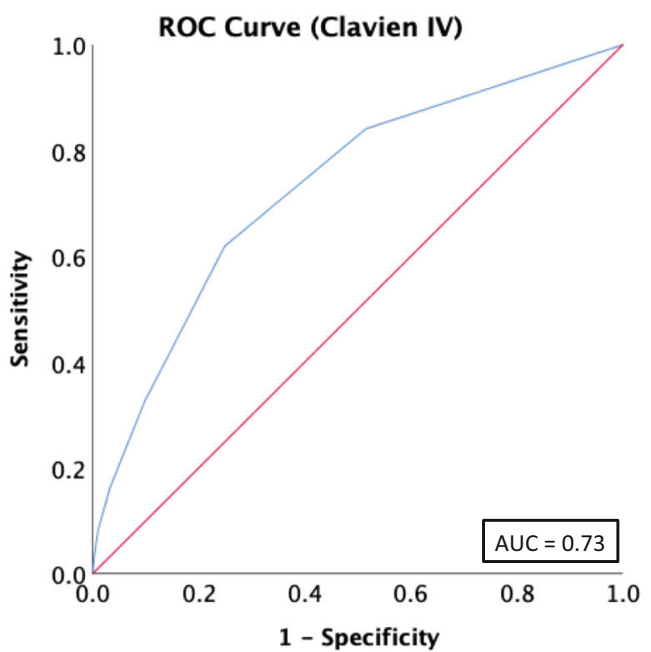

Fig. 1 Receiver operator characteristic curves (ROC) of modified frailty index $(\mathrm{mFI})$ for a mortality and $\mathbf{b}$ Clavien IV complications

acute cholecystitis. Frailty is associated with an increased risk for death up to 12 -fold for patients with high-frailty. Confirmed by ROC analysis, $\mathrm{mFI}$ is sensitive and specific for predicting postoperative CLIVCs (AUC 0.73) and mortality (AUC 0.83) (Fig. 1). Additionally, individual frailty components were studied and demonstrated that dependent functional status carried the most risk in contributing to postoperative morbidity (OR 4.28, 95\% CI 3.21-5.71, $p<0.001$ ) and mortality (OR 9.28, 95\% CI 5.81-14.8, $p<0.001$ ) (Tables 5 and 6). Studying individual frailty components is important to generate procedure-specific variables that may be utilized in future frailty scoring systems.

The application of $\mathrm{mFI}$ using the NSQIP database has allowed for the use of a prognostic model to a heterogeneous population for a single disease process to measure clinical outcomes. Frailty is a marker for the sum of overall physiologic status and a useful tool to determine the risk of postoperative complications. Multiple models exist for calculating frailty, including the Frailty Index of Cumulative Deficits, Fried's Frailty Phenotype, and the Frailty Index of Accumulative Deficit. ${ }^{20}$ These models have been noted to have limitations as they are burdened by either increasing complexity or too simplistic which translates to limited predictability. Despite the increasing variety of frailty models, there is no universally accepted frailty definition or components. However, the mFI strikes a reasonable balance and has demonstrated clinical utility in colorectal, pancreatic, hepatic, vascular, and gynecologic surgery. ${ }^{15,21-23}$ Recently, Murphy and colleagues have studied the impact of frailty on outcomes for patients undergoing various emergency general surgical procedures using mFI. In their analysis, $14 \%$ of patients underwent a cholecystectomy, and high frail patients were more likely to suffer a serious complications (OR 2.7, 95\% CI 1.7 6.3 ) and were less likely to be discharged home (OR 0.25 , 95\% CI 0.19-0.33). Our study corroborates these findings in patients undergoing non-emergent cholecystectomy.

Patients with low frailty had an increased risk of CLIVCs (OR 1.73, 95\% CI 1.15-2.61, $p=0.009$ ) but no increased risk of mortality (OR 1.44, 95\% CI $0.51-4.10, p=0.494$ ). Enhanced risk stratification using the $\mathrm{mFI}$, which can detect those with intermediate and high frailty, can allow patients to have their comorbidities optimized. These patients might be treated with intravenous antibiotics and placement of percutaneous cholecystostomy tube. Following medical therapy, frail patients can undergo preoperative rehabilitation known as "prehab," which has shown to improve surgical outcomes. ${ }^{24,25}$ Thus, the mFI is a useful tool for preoperative risk stratification that affects clinical decision making.

This study highlights the importance of individual frailty components and their risk towards postoperative morbidity and mortality. In fact, the 2018 Tokyo guidelines has added more patient specific factors including neurological dysfunction, ASA, and CCI to improve its accuracy. ${ }^{12}$ On the other hand, the $\mathrm{mFI}$ has recently decreased the number of variables from 11 to 5, including the omission of impaired sensorium. In our study, impaired sensorium was a strong predictor of mortality (OR 4.34, 95\% CI 2.25-8.38, $p<0.001$ ) (Table 6). We believe that the decrease in the number of frailty components from 11 to 5 may diminish the predictive accuracy. Furthermore, a weighted frailty risk score incorporating the individual frailty components might produce the best procedure-specific risk score.

Age is often utilized as a surrogate for frailty and to identify high-risk patients. However, data is conflicted with respect to age and outcomes following cholecystectomy. ${ }^{26-28}$ Kanakala and colleagues analyzed 2117 patients undergoing LC from the UK at a single institution and found no association between increasing age and complications following $\mathrm{LC}^{26}$ 
Conversely, Murphy et al. utilized the National Inpatient Sample and studied 1,102,071 patients from 1998 to 2006 and found advanced age ( $>65$ years old) to be associated with increased complications (OR 2.16, 95\% CI 2.01-2.32) and conversion to an open procedure (OR 2.61, 95\% CI $2.42-$ $2.81){ }^{27}$ Furthermore, Giger et al. studied 22,953 patients undergoing LC from a Swiss national database and found increased age to be associated with increased rate of intraoperative and postoperative local/systemic complications (all $p<0.001){ }^{28}$ Our multivariable regression revealed that age was not associated with an increased rate of CLIVCs (OR $1.16,95 \%$ CI $0.58-2.29, p=0.551$ ) or mortality (OR 2.24, 95\% CI 0.62-8.07, $p=0.217$ ) (Tables 3 and 4).Instead, increasing frailty was significantly $(p<0.001)$ associated with postoperative CLIVCs and mortality (Tables 3 and 4). This suggests that with the advances in perioperative care, older patients can undergo laparoscopic cholecystectomy without an increased risk of complications and mortality.

This study is not without limitations. While NSQIP variables are collected in a prospective fashion, the $\mathrm{mFI}$ was retrospectively applied to patients which may lead to misclassification error. Percutaneous cholecystostomy tube placement data are not collected by NSQIP and therefore no comparison can be made to patients undergoing this procedure. In addition, we are unable to identify those patients who undergo cholecystectomy after percutaneous drainage. We also acknowledge that some of the variables in the $\mathrm{MFI}$ are no longer collected in NSQIP. We argue they should be brought back in, especially impaired sensorium which carried an increased risk of postoperative mortality.

\section{Conclusion}

In summary, this study demonstrates the association of frailty with postoperative morbidity and mortality in patients undergoing laparoscopic cholecystectomy for acute cholecystitis. Increasing frailty however is significantly associated with Clavien IV complications and mortality while age is not. The mFI should be considered as a tool to aid in risk stratification and clinical decision making for patients who are traditionally thought to be high risk or the elderly. Individualized assessment of patients by frailty components may assist in the decision to pursue cholecystectomy or an alternative treatment option. Future research is needed to corroborate the findings in this retrospective cohort study.

Author Contribution Design of the work: AF, BP, KL

Analysis and interpretation of data for the work: AF, BP, KZ, KL

Drafting of the work: AF, KZ

Critical revision: AF, BP, KZ, SK, AK, ADC, KL

Final approval of the version to be published: AF, BP, KZ, SK, AK, $\mathrm{ADC}, \mathrm{KL}$
Agreement to be accountable for all aspects of the work in ensuring that all questions related to the accuracy or integrity of any part of the work are appropriately investigated and resolved: all authors

\section{Compliance with Ethical Standards}

Conflict of Interest The authors declare that they have no conflict of interest.

\section{References}

1. United States. Agency for Healthcare Research and Quality. Healthcare Cost and Utilization Project (HCUP) statistical briefs. In: Trends in Operating Room Procedures in U.S. Hospitals, 20012011. Rockville, MD: Agency for Health Care Policy and Research,; 2006: http://www.ncbi.nlm.nih.gov/books/NBK52651. Accessed 30 Jul 2019.

2. Winbladh A, Gullstrand P, Svanvik J, et al. Systematic review of cholecystostomy as a treatment option in acute cholecystitis. HPB (Oxford). 2009;11:183-193.

3. Tsuyuguchi T, Itoi T, Takada T, et al. TG13 indications and techniques for gallbladder drainage in acute cholecystitis. J Hepatobiliary Pancreat Sci. 2013;20:81-88.

4. Viste A, Jensen D, Angelsen JH, et al. Percutaneous cholecystostomy in acute cholecystitis; a retrospective analysis of a large series of 104 patients. BMC Surg. 2015;8:15-17.

5. Baron TH, Topazian MD. Endoscopic transduodenal drainage of the gallbladder: implications for endoluminal treatment of gallbladder disease. Gastrointest Endosc. 2007;65:735-737.

6. Boregowda U, Umapathy C, Nanjappa A, et al. Endoscopic ultrasound guided gallbladder drainage - is it ready for prime time? World J Gastrointest Pharmacol Ther. 2018;9:47-54.

7. McGregor H, Woodhead G, Conrad M. et al. First In-Human Gallbladder Cryoablation in a Patient with Acute Calculous Cholecystitis Initially Treated with a Cholecystostomy Tube. J Vasc Interv Radiol. 2019;18:31780-31789.

8. Ansaloni L, Pisano M, Coccolini F, et al. 2016 WSES guidelines on acute calculous cholecystitis. World J Emerg Surg. 2016;14:11-25.

9. Saito R, Abe T, Hanada K, et al. Impact of comorbidities on the postoperative outcomes of acute cholecystitis following early cholecystectomy. Surg Today. 2017;47:1230-1237

10. Karamanos E, Sivrikoz E, Beale E, et al. Effect of diabetes on outcomes in patients undergoing emergent cholecystectomy for acute cholecystitis. World J Surg. 2013;37:2257-2264.

11. Murphy PB, Savage SA, Zarzaur BL. Impact of Patient Frailty on Morbidity and Mortality after Common Emergency General Surgery Operations. J Surg Res. 2019;S0022-4804(19)1-8.

12. Yokoe M, Hata K, Takada T, et al. Tokyo Guidelines 2018: diagnostic criteria and severity grading of acute cholecystitis (with videos). J Hepatobiliary Pancreat Sci. 2018;25:41-54.

13. Rockwood K, Song X, MacKnight C, et al. A global clinical measure of fitness and frailty in elderly people. CMAJ. 2005;173:489495.

14. Velanovich V, Antoine H, Swartz A, et al. Accumulating deficits model of frailty and postoperative mortality and morbidity: its application to a national database. J Surg Res. 2013;183:104-110.

15. Louwers L, Schnickel G, Rubinfeld I. Use of a simplified frailty index to predict Clavien 4 complications and mortality after hepatectomy: analysis of the National Surgical Quality Improvement Project database. Am J Surg 2016;211:1071-1076.

16. Makary MA, Segev DL, Pronovost PJ, et al. Frailty as a predictor of surgical outcomes in older patients. J Am Coll Surg. 2010;210:901908 . 
17. Dasgupta M, Rolfson DB, Stolee P, et al. Frailty is associated with postoperative complications in older adults with medical problems. Arch Gerontol Geriatr. 2009;48:78-83.

18. Saxton A, Velanovich V. Preoperative frailty and quality of life as predictors of postoperative complications. Ann Surg. 2011;253: 1223-1229.

19. Obeid NM, Azuh O, Reddy S, et al. Predictors of critical carerelated complications in colectomy patients using the National Surgical Quality Improvement Program: exploring frailty and aggressive laparoscopic approaches. J Trauma Acute Care Surg. 2012;72:878-883.

20. Fried LP, Ferrucci L, Darer J, et al. Untangling the concepts of disability, frailty, and comorbidity: implications for improved targeting and care. J Gerontol A Biol Sci Med Sci. 2004;59:255263.

21. Augustin T, Burstein MD, Schneider EB, et al. Frailty predicts risk of life-threatening complications and mortality after pancreatic resections. Surgery. 2016;160:987-996.

22. Karam J, Tsiouris A, Shepard A, et al. Simplified frailty index to predict adverse outcomes and mortality in vascular surgery patients. Ann Vasc Surg. 2013;27:904-908.

23. Uppal S, Igwe E, Rice LW, et al. Frailty index predicts severe complications in gynecologic oncology patients. Gynecol Oncol. 2015;137:98-101.
24. Partridge JSL, Harari D, Martin FC, et al. Randomized clinical trial of comprehensive geriatric assessment and optimization in vascular surgery. Br J Surg. 2017;104:679-687.

25. Harari D, Hopper A, Dhesi J, et al. Proactive care of older people undergoing surgery ('POPS'): designing, embedding, evaluating and funding a comprehensive geriatric assessment service for older elective surgical patients. Age Ageing, 2007;36;190-196.

26. Kanakala V, Borowski DW, Pellen MG, et al. Risk factors in laparoscopic cholecystectomy: a multivariable analysis. Int J Surg. 2011;9:318-323.

27. Murphy MM, Ng SC, Simons JP et al. Predictors of major complications after laparoscopic cholecystectomy: surgeon, hospital, or patient. J Am Coll Surg. 2010;211:73-80.

28. Giger UF, Michel JM, Opitz I, et al. Risk factors for perioperative complications in patients undergoing laparoscopic cholecystectomy: analysis of 22,953 consecutive cases from the Swiss Association of Laparoscopic and Thoracoscopic Surgery database. J Am Coll Surg 2006;203:723-728.

Publisher's Note Springer Nature remains neutral with regard to jurisdictional claims in published maps and institutional affiliations. 